\title{
CARBON STAR DUST FROM METEORITES
}

\author{
UFFE GRÅE JØRGENSEN AND ANJA C. ANDERSEN \\ Niels Bohr Institute, Copenhagen University Observatory \\ Copenhagen, Denmark
}

\begin{abstract}
Inside carbonaceous chondrite meteorites are tiny dust particles which, when heated, release noble gases with an isotopic composition different from what is found anywhere else in the solar system. For this reason it is believed that these grains are (inter)stellar dust which survived the collapse of the interstellar cloud that became the solar system. We will describe here why we believe that the most abundant of these grains, microdiamonds, were formed in the atmospheres of carbon stars, and explain how this theory can be tested observationally.
\end{abstract}

\section{Introduction}

The discovery of meteoritic dust grains with origin outside the solar system has opened the possibility of studying presolar material in the laboratory, with all the advantages in details and accuracy such analyses allow for. Identification of the (possible) stellar origin of the meteoritic grains offers a unique opportunity to add important new constrains on models of stellar evolution (detailed elemental and isotopic abundances) and stellar atmospheric structure (elemental and mineralogical composition of the grains). There are several indications that a fraction, possibly the bulk, of the presolar meteoritic grains has its origin in carbon stars.

For the moment the amount of detailed information (such as isotopic ratios of tiny noble gas impurities) about the meteoritic grains is overwhelming (see e.g. Zinner 1995), whereas much of the fundamental data necessary in order to apply the meteoritic results to stellar modelling is entirely missing. For example, the necessary rate coefficients for formation of the most abundant presolar meteoritic grains (diamond dust) are lacking, and stellar wind models therefore do not predict diamond formation, but instead such models predict amorphous carbon (which has not been identified in meteorites) as the most abundant grain type in carbon-rich environments. 
A combined self-consistent description of the full atmospheric region of a red giant star does not yet exist, but is slowly coming within reach. The meteoritic data combined with more fundamental laboratory data can be an important ingredient in constructing such a model for the first time. Successful construction of self-consistent models, followed possibly by verification of the formation place(s) of the most abundant stellar grains that contributed to the formation of the meteorites (and hence also the planets), would provide fundamental new knowledge about the sources of material for the solar system and the chemical evolution of the Galaxy.

The most common types of meteorites are fragments of larger protoplanetary bodies, which melted and chemically differentiated after their formation. Carbonaceous chondrites, on the other hand, are meteorites which have never been part of a larger body. They consist of spherical glass-like chondrules embedded in a fine-grained matrix. The matrix has had a gentle thermal history and is believed to be the (relatively unprocessed) original dust from which the planets formed. Therefore, the larger the amount of matrix in the chondrite, relative to the chondrule material, the more original solar nebula material is present, and the more primitive the chondrite is said to be.

When this matrix material was heated in the laboratory, it was realized already in the early 1960's (see Lewis \& Anders 1983 for a review) that at certain temperatures the matrix released noble gases with an isotopic composition markedly different from everything else in the solar system. It was therefore concluded that the matrix contains one or more types of grains, formed before the solar system, in which non-solar-composition noble gases are trapped. After years of trials with different chemical purifications of the matrix material, and subsequent stepwise heating and isotopic noble gas measurements, the first presolar grains were finally isolated by Lewis et al. in 1987, and were identified as tiny diamonds.

Diamonds account for more than $99 \%$ of the identified presolar meteoritic material, with an abundance that can exceed $0.1 \%(1000 \mathrm{ppm})$ of the matrix (Huss \& Lewis 1994b), corresponding to more than $3 \%$ of the total amount of carbon in the meteorite. The second and third most abundant types are $\mathrm{SiC}(6 \mathrm{ppm})$ and graphite (less than $1 \mathrm{ppm})$. They are all chemically quite resistant, which makes it possible to isolate them by dissolving the meteorite in acids. Further, a few of the $\mathrm{SiC}$ and graphite grains have been shown to contain tiny sub-grains of titanium and refractory carbides (Bernatowicz et al. 1991, 1992, 1994). Three isotopically anomalous, noncarbon-bearing grains have also been found. They are corundum $\left(\mathrm{Al}_{2} \mathrm{O}_{3}\right)$, spinel $\left(\mathrm{MgAl}_{2} \mathrm{O}_{4}\right)$, and silicon nitride $\left(\mathrm{Si}_{3} \mathrm{~N}_{4}\right)$ (Russel et al. 1995, Nittler et al. 1994, 1995). In the following sections we will discuss diamonds, $\mathrm{SiC}$ and graphite in some detail. 


\section{Diamonds}

The individual diamond grains are very small, with a median diameter of less than $20 \AA$ (Fraundorf et al. 1989). Since the diamond lattice distance is about $2 \AA$, a typical presolar diamond contains of the order $\left(\frac{20}{2}\right)^{3}=$ 1000 carbon atoms, with $6 \times 10^{2} \approx 50 \%$ of these belonging to the surface. Since surface atoms have one unpaired bond, they will (in a hydrogen-rich atmosphere) resemble hydrogenated amorphous carbon (a-C:H). Only the $\sim 50 \%$ "interior" atoms will sit in an actual diamond crystal structure. The presolar diamonds are therefore often called amorphous diamonds.

It is not obvious to what degree the extracted diamonds resemble the original diamond dust at its place of origin. Many alterations could have occurred in interstellar space or in the solar nebula, as well as during the chemical extraction process in the laboratory. However, the first step in an observational identification of their astronomical source of origin might be to model their synthetic spectrum. For this purpose we have measured the monochromatic absorption coefficient, described which of the features can be expected to be intrinsic to the diamonds (and which might be artifacts from the chemical processing in the laboratory), and computed synthetic carbon star spectra with the diamonds included (Andersen et al. 1998). The features which are most likely to be intrinsic are listed in Table 1 and compared with the results obtained by other groups.

As for the other grains, the strongest argument that the diamonds are formed outside the solar system is the peculiar, non-solar isotopic composition of their noble gas inclusions (and other trace element inclusions). There are several reasons why we believe the bulk of the diamonds formed in carbon stars, one of the most important being their ${ }^{12} \mathrm{C} /{ }^{13} \mathrm{C}$ ratio of $\sim 90$. This ratio is identical to what is observed in carbon stars with a large excess of carbon (i.e. with $\mathrm{C} / \mathrm{O} \gtrsim 1.5$, and strongly mass-losing), and it is not found in any other abundant astronomical objects. In contrast to this, $\mathrm{SiC}$ (the second most abundant presolar grain) has ${ }^{12} \mathrm{C} /{ }^{13} \mathrm{C} \approx 40$, which is typical (Lambert et al. 1986) for carbon stars with only small excesses of carbon (i.e. with $\mathrm{C} / \mathrm{O} \approx 1$ ). Hydrostatic MARCS photospheric models indicate that $\mathrm{SiC}$ grains will dominate the grain formation for $\mathrm{C} / \mathrm{O} \approx 1$ (where ${ }^{12} \mathrm{C} /{ }^{13} \mathrm{C}$ is as found in the meteoritic $\mathrm{SiC}$ grains), whereas pure carbon grains will dominate for the high $\mathrm{C} / \mathrm{O}$ ratios (where ${ }^{12} \mathrm{C} /{ }^{13} \mathrm{C}$ is as in the meteoritic diamonds). This was the primary basis for our theory (Jørgensen 1988) that diamonds come from evolved carbon stars and $\mathrm{SiC}$ from less evolved carbon stars (actually, at the time the paper was written it was our prediction that $\mathrm{SiC}$ should exist in meteorites). The (radiative pressure driven) mass loss increases rapidly with increasing $\mathrm{C} / \mathrm{O}$ (= increasing ${ }^{12} \mathrm{C} /{ }^{13} \mathrm{C}$ ) of the stars. If diamonds and $\mathrm{SiC}$ are formed in carbon stars, it is therefore a natural 
TABLE 1. Spectral features, in $\mathrm{cm}^{-1}$, detected in the spectra of presolar diamonds from the Allende, Murchison and Orgueil meteorites.

\begin{tabular}{|c|c|c|c|c|c|c|}
\hline \multicolumn{3}{|c|}{ ALLENDE } & \multicolumn{2}{|c|}{ MURCHISON } & \multirow{2}{*}{$\frac{\text { ORGUEIL }}{(6)^{a}}$} & \multirow[t]{2}{*}{ ASSIGNMENT } \\
\hline \multirow[t]{3}{*}{ (1) } & $(2)^{a}$ & (3) & (4) & (5) & & \\
\hline & & 50000 & & 50000 & & paired $\mathrm{N}$ in diamond \\
\hline & & 37037 & & 37037 & & paired $\mathrm{N}$ in diamond \\
\hline 2919 & & 2954 & & 3000 & 2940 & aliphatic $\mathrm{C}-\mathrm{H}$ stretch \\
\hline 2849 & & 2854 & & 2800 & & \\
\hline 1361 & & 1385 & 1399 & & 1380 & $\begin{array}{l}\mathrm{C}-\mathrm{H} \text { deformation }\left(\mathrm{CH}_{3}\right) \\
\text { interstitial } \mathrm{N}\end{array}$ \\
\hline 1173 & 1143 & 1122 & 1084 & 1175 & 1042 & $\mathrm{C}-\mathrm{O} / \mathrm{C}-\mathrm{N}$ stretch \\
\hline \multirow[t]{5}{*}{1028} & 1089 & 1054 & & 1090 & & $\mathrm{CH}_{2}$ waging \\
\hline & 626 & & & & 620 & $\mathrm{CH}$ out-of-plane \\
\hline & & & & 396,367 & & $\mathrm{C}=\mathrm{O}=\mathrm{C}$ or $\mathrm{C}=\mathrm{N}=\mathrm{C}$ \\
\hline & & & & 310 & & \\
\hline & & & & 130,120 & & ?? \\
\hline
\end{tabular}

(1) Lewis et al. 1989, (2) Koike et al. 1995, (3) Andersen et al. 1998,

(4) Lewis 1992, (5) Mutschke et al. 1996, (6) Wdowiak et al. 1988

${ }^{a}$ the spectra were obtained on diamond-like residues

consequence of this theory that the meteorites contain much more diamonds than SiC. A more quantitative simulation is still lacking because at present it isn't possible to include diamond formation in the model atmospheres (due to lack of basic input data).

A number of impurities have been identified in the presolar diamonds, including the noble gases ( $\mathrm{He}, \mathrm{Ne}, \mathrm{Ar}, \mathrm{Kr}$, and $\mathrm{Xe}$ ), $\mathrm{Ba}$ and $\mathrm{Sr}$ (which are slightly enriched in $r$-process isotopes; Lewis et al. 1991), $\mathrm{H}$ with ${ }^{1} \mathrm{H} /{ }^{2} \mathrm{D}$ $=5193\left(\right.$ Virag et al. $\left.1989 ;\left({ }^{1} \mathrm{H} /{ }^{2} \mathrm{D}\right)_{\text {terrestial }}=6667\right)$ and $\mathrm{N}$ with ${ }^{14} \mathrm{~N} /{ }^{15} \mathrm{~N}$ $=406$ (Russel et al. 1991; $\left.\left({ }^{14} \mathrm{~N} /{ }^{15} \mathrm{~N}\right)_{\text {terrestial }}=272\right)$. The most important of these, $\mathrm{Xe}$, was actually known from stepwise heating techniques before the grains themselves were identified as diamonds. The Xe in the diamonds has a significant overabundance (compared to the solar isotopic ratios) of the very heavy isotopes (Xe-H, i.e. isotopes ${ }^{134} \mathrm{Xe}$ and ${ }^{136} \mathrm{Xe}$ ) as well as the very light isotopes (Xe-L, i.e. ${ }^{124} \mathrm{Xe},{ }^{126} \mathrm{Xe}$ ). This composition is often called Xe-HL to indicate that there is an excess of both heavy $(\mathrm{H})$ and light (L) isotopes. There are no astronomical objects known (either from observation or from standard theories) which have both a solar ${ }^{12} \mathrm{C} /{ }^{13} \mathrm{C}$ ratio and Xe-HL. An explanation therefore needs to involve either a nonstandard model, not yet observationally verified, or an assumption of the diamonds being a mixture of populations from several different sources.

Heavy and light Xe isotopes are produced in supernovae (SN), and Clay- 
ton (1989) therefore proposed that the meteoritic diamond grains were formed in a supernova that also produced the Xe-HL measured in the diamonds. Since the progenitor star of a supernova in the standard theories has an oxygen-rich atmosphere (i.e., cannot produce carbon-rich grains) and a pure ${ }^{12} \mathrm{C}$ interior shell (i.e., can only produce grains with ${ }^{13} \mathrm{C} /{ }^{12} \mathrm{C}$ $\approx 0$ ), a non-standard theory was necessary. In an extension of the model, Clayton et al. (1995) proposed a non-standard SN where mixing from a ${ }^{13} \mathrm{C}$-rich shell occurs in the right amount to give ${ }^{12} \mathrm{C} /{ }^{13} \mathrm{C} \approx 90$. A nonstandard $r$-process was assumed too, in order to avoid the production of ${ }^{129} \mathrm{I}$ which decays to ${ }^{129} \mathrm{Xe}$ and would therefore cause a very large excess of ${ }^{129} \mathrm{Xe}$, not observed in the meteorites. For a recent review of the standard $r$ - and $s$ - neutron capture processes, see Käppeler et al. (1989). Furthermore, the regular $r$-process cannot in itself produce the very large excess of ${ }^{136} \mathrm{Xe}$ characteristic of the Xe-HL measured in presolar diamonds. Ott (1996), however, proposed that the standard $r$-process is active, but that a separation of xenon from iodine and tellurium precursors takes place in SN on a time scale of a few hours after termination of the neutron burst in the SN. Since ${ }^{136} \mathrm{Xe}$ is formed minutes after the neutron burst, and the other $r$-process Xe isotopes are formed hours $\left({ }^{134} \mathrm{Xe}\right)$, days $\left({ }^{131} \mathrm{Xe}\right)$, or even years $\left({ }^{129} \mathrm{Xe}\right)$ later, a sufficiently early separation would allow almost infinite amounts of ${ }^{136} \mathrm{Xe}$ relative to the other Xe isotopes which are produced. If a separation in the $\mathrm{SN}$ gas takes place two hours after the neutron burst, the meteoritic ${ }^{136} \mathrm{Xe} /{ }^{134} \mathrm{Xe}$ ratio is established in the gas, and with a small amount of later mixing, the observed meteoritic $\mathrm{Xe}-\mathrm{H}$ can be obtained.

Detailed supernova models supporting these isotopic arguments are lacking (as are simulations justifying, for example, the amount of ${ }^{13} \mathrm{C}$ mixing or why only Xe from the separated gas is implanted in the grains when they form years after the neutron burst, etc.), but the success of fitting modified SN scenarios to the observed Xe-H makes it likely that part of the diamond grains originates in supernovae. However, there are several reasons why the bulk of the diamonds are unlikely to have formed in supernovae: (1) The hydrodynamical time scale of a supernova is short compared to the time scale for carbon grain formation (Sedlmayr 1994). (2) The mass loss is much stronger in carbon stars with high $\mathrm{C} / \mathrm{O}$ ratios (where pure carbon grains will form) than in carbon stars with lower $\mathrm{C} / \mathrm{O}$ ratios. If the $\mathrm{SiC}$ is formed in carbon stars (see next section), there will therefore have been expelled much more diamond dust from carbon stars (or other pure carbon grains, which are, however, not seen) into interstellar space than SiC. (3) Carbon stars were very abundant in the Galaxy prior to the formation of the solar system (due to their metallicity dependence). The resemblance of the carbon star ${ }^{12} \mathrm{C} /{ }^{13} \mathrm{C}$ to the solar ${ }^{12} \mathrm{C} /{ }^{13} \mathrm{C}$ is naturally explained if carbon stars were the source of the solar carbon (including carbon grains), 
whereas the standard $\mathrm{SN}$ not will produce this ${ }^{12} \mathrm{C} /{ }^{13} \mathrm{C}$ ratio.

The typical amount of Xe gas inclusion in the diamonds is $\sim 10^{-6} \mathrm{~cm}^{3}$ per gram of diamond. This corresponds to approximately one ${ }^{132} \mathrm{Xe}$ atom and one ${ }^{129} \mathrm{Xe}$ atom per $10^{7}$ diamonds, a bit less of the isotopes ${ }^{131} \mathrm{Xe}$, ${ }^{134} \mathrm{Xe}$, and ${ }^{136} \mathrm{Xe}$, one-tenth this amount of isotopes ${ }^{128} \mathrm{Xe}$ and ${ }^{130} \mathrm{Xe}$, and only traces of ${ }^{124} \mathrm{Xe}$ and ${ }^{126} \mathrm{Xe}$. A large number of diamonds is therefore necessary in order to perform an isotopic analysis (in practice $\sim 10^{10}$, Huss \& Lewis 1994a), and attempts to separate diamonds into groups of different origin have so far not been successful (Huss \& Lewis 1994b). If we assume that the trapping efficiency for Xe in the possible population of diamonds which originated in supernovae is sufficiently large compared to the trapping efficiency in the carbon star diamonds (perhaps because of the higher turbulent gas velocities in $\mathrm{SN}$, higher densities, etc.), then the $\mathrm{Xe}-\mathrm{H}$ can be explained as being connected with a small fraction of diamonds of pure ${ }^{12} \mathrm{C}$ (which originated in $\mathrm{SN}$ ), without altering the necessary bulk ${ }^{12} \mathrm{C} /{ }^{13} \mathrm{C}$ ratio of the carbon star diamonds. We therefore propose that the bulk of the presolar meteoritic diamonds originates in evolved carbon stars (as in our original theory) and are mixed with a smaller population (from SN II) which has a relatively high Xe content and is rich in the heavy isotopes. The content of light isotopes of Xe is very small (less than 1 atom per $10^{9}$ diamonds) and can be explained as coming from SN I (Lambert 1992) in binary systems where the low-mass component is an evolved carbon star as in our original theory (Jørgensen 1988), or as a by-product of the Xe production in SN II (Ott 1996).

\section{Silicon Carbide}

$\mathrm{SiC}$ is much less abundant (6 ppm) than diamonds (1000 ppm), but some of the $\mathrm{SiC}$ grains are large enough that isotopic ratios of $\mathrm{Si}$ and $\mathrm{C}$ (and the abundant impurities $\mathrm{N}, \mathrm{Mg}-\mathrm{Al}, \mathrm{Ti}, \mathrm{Ca}, \mathrm{He}, \mathrm{Ne}$ ) can be measured in individual grains (Hoppe et al. 1994; Lewis et al. 1994; Anders \& Zinner 1993 , and references therein). The understanding of their stellar origin is therefore much better than in the case of the diamonds.

The SiC grain sizes have a large variety from less than 0.05 to $20 \mu \mathrm{m}$ in equivalent spherical diameter, with about $95 \%$ (by mass) of the grains being between 0.3 and $3 \mu \mathrm{m}$ (Amari et al. 1994). Ion micro-probe measurements can be performed on individual grains larger than $1 \mu \mathrm{m}$, and the results have made it possible to identify multiple stellar sources as their origin. The detailed match to the elemental and isotopic conditions in the He burning shell of AGB stars (Gallino et al. 1990) has made it generally believed that the bulk of the $\mathrm{SiC}$ originated in carbon stars.

In order to distinguish between various stellar origins, Hoppe et al. 
(1994) have divided the coarse $(2.1-5.9 \mu \mathrm{m}) \mathrm{SiC}$ grains into five subgroups:

1. The "mainstream" grains have $20<{ }^{12} \mathrm{C} /{ }^{13} \mathrm{C}<120$ and $200<{ }^{14} \mathrm{~N} /{ }^{15} \mathrm{~N}<10000$.

2. Grains A have ${ }^{12} \mathrm{C} /{ }^{13} \mathrm{C}<3.5$.

3. Grains $\mathrm{B}$ have $3.5<{ }^{12} \mathrm{C} /{ }^{13} \mathrm{C}<10$.

4. Grains $X$ have isotopically heavy $\mathrm{N}\left(13<{ }^{14} \mathrm{~N} /{ }^{15} \mathrm{~N}<180\right)$.

5. Grains $\mathrm{Y}$ have isotopically light $\mathrm{C}\left(150<{ }^{12} \mathrm{C} /{ }^{13} \mathrm{C}<260\right)$.

The mainstream, type A, and type B grains have comparable patterns of Si isotopes, distinctly different from type $\mathrm{X}$ and type $\mathrm{Y}$ grains. The mainstream grains constitute $\sim 94 \%$ of all coarse-grained $\mathrm{SiC}$, whereas grains from the groups A, B, X and Y account for only $2 \%, 2.5 \%, 1 \%$ and $1 \%$, respectively. Based on this grouping, Amari et al. (1995a) find that grains X could originate from a supernova (SN II), and Lodders \& Fegley (1995) find that grains $\mathrm{A}$ and $\mathrm{B}$ can be at least qualitatively understood if they originate from J-type carbon stars or carbon stars that have not experienced much dredge-up of He-shell material.

It is seen that the isotopic variations among the grains are very large. The ${ }^{12} \mathrm{C} /{ }^{13} \mathrm{C}$ ratio varies by a factor of more than 350 , and ${ }^{14} \mathrm{~N} /{ }^{15} \mathrm{~N}$ varies by 300 times (and ${ }^{30} \mathrm{Si} /{ }^{28} \mathrm{Si}$ by a factor of 3 ). Variations in noble gases associated with $\mathrm{SiC}$ are large as well (Ott 1993). The two noble gas components, $s$-process Xe and neon-E (i.e., essentially pure ${ }^{22} \mathrm{Ne}$ ), show opposite correlations with grain size, $s$-process Xe being most abundant in fine-grained $\mathrm{SiC}$ and $\mathrm{Ne}-\mathrm{E}$ in coarse-grained $\mathrm{SiC}$ (Lewis et al. 1994). Other elements which show $s$-process indications include $s$-process $\mathrm{Kr}, \mathrm{Ba}, \mathrm{Sr}, \mathrm{Ca}, \mathrm{Ti}, \mathrm{Nd}$ and Sm.

The proportions of ${ }^{80,86} \mathrm{Kr}$ vary with release temperature of the gas. This variation reflects branching of the $s$-process at the radioactive progenitors, ${ }^{79} \mathrm{Se}$ and ${ }^{85} \mathrm{Kr}$ (Ott et al. 1988). These branchings depend sensitively on neutron density and temperatures in the $s$-process region, and the ${ }^{80,86} \mathrm{Kr}$ can therefore provide clues about in which stars the $\mathrm{SiC}$ formed, or if the stellar type is already known it can put constraints on the detailed modelling of these stars.

\section{Graphite}

The presolar graphite isolated from meteorites lies at the graphitic end of the continuum between kerogen, amorphous carbon, and graphite. It is not very abundant (less than $1 \mathrm{ppm}$ ), and it is much more complicated to extract than SiC and micro-diamonds (Amari et al. 1994). Presolar graphite occurs solely in the form of spherules, $0.8-8 \mu \mathrm{m}$ in diameter, while graphite grains of other sizes and shapes have normal composition and are believed to have been formed in the solar nebula (Zinner et al. 1990). The presolar 
graphite has a very broad ${ }^{12} \mathrm{C} /{ }^{13} \mathrm{C}$ distribution, with ${ }^{12} \mathrm{C} /{ }^{13} \mathrm{C}$ ratios ranging from 7 to 4500 , whereas the ${ }^{14} \mathrm{~N} /{ }^{15} \mathrm{~N}$ ratios range from 193 to 680 (Zinner et al. 1995).

The noble gases show systematic trends with sample density, suggesting more than one kind of graphite. Some have almost mono-isotopic ${ }^{22} \mathrm{Ne}$. Others contain neon with a somewhat higher ${ }^{20} \mathrm{Ne} /{ }^{22} \mathrm{Ne}$ ratio and are accompanied by $s$-process $\mathrm{Kr},{ }^{4} \mathrm{He}$, and other noble gases (Amari et al. 1995b).

The carbon and nitrogen isotopic ratios found in the grains indicate that they come from stellar sources dominated by H-burning rather than from sources dominated by He-burning (Amari et al. 1993). H-burning in the CNO cycle produces isotopically heavy carbon $\left({ }^{13} \mathrm{C}\right)$ and light nitrogen $\left({ }^{14} \mathrm{~N}\right)$, in qualitative agreement with the measurements (Zinner et al. 1989; Hoppe et al. 1994). Systematic measurements of isotopic ratios of several other elements were recently done by Hoppe et al. (1995).

\section{References}

Amari, S., Hoppe, P., Zinner, E. \& Lewis, R. S. 1993, Nature, 365, 806

Amari, S., Hoppe, P., Zinner, E. \& Lewis, R.S. 1995a, Meteoritics, 30, 679

Amari, S., Lewis, R. S. \& Anders, E. 1994, Geochim. Cosmochim. Acta, 58, 459

Amari, S., Lewis, R.S. \& Anders, E. 1995b, Geochim. Cosmochim. Acta, 59, 1411

Anders, E. \& Zinner, E. 1993, Meteoritics, 28, 490

Andersen, A. C., Jørgensen, U. G., Nicolaisen, F. M., Sørensen, P. G. \& Glejbøl, K. 1998, $A \& A, 330,1080$

Bernatowicz, T. J., Amari, S. \& Lewis, R.S. 1992, Lunar Planet. Sci., 23, 91

Bernatowicz, T. J., Amari, S. \& Lewis, R.S. 1994, Lunar Planet. Sci., 25, 103

Bernatowicz, T. J., Amari, S., Zinner, E. \& Lewis, R. S. 1991, ApJ, 373, L73

Clayton, D. D. 1989, ApJ, 340, 613

Clayton, D. D., Mayer, B.S., Sanderson, C. I., Russel, S.S. \& Phillinger, C. T. 1995, ApJ, 447,894

Fraundorf, P., Fraundorf, G., Bernatowicz, T., Lewis, R.S. \& Tang, M. 1989, Ultramicroscopy, 27, 401

Gallino, R., Busso, M., Picchio, G. \& Raitari, C. M. 1990, Nature, 348, 298

Hoppe, P., Amari, S., Zinner, E., Ireland, T. \& Lewis, R. S. 1994, ApJ, 430, 870

Hoppe, P., Amari, S., Zinner, E. \& Lewis, R. S. 1995, Geochim. Cosmochim. Acta, 59, 4029

Huss, G.R. \& Lewis, R.S. 1994a, Meteoritics, 29, 791

Huss, G.R. \& Lewis, R. S. 1994b, Meteoritics, 29, 811

Jørgensen, U. G. 1988, Nature, 332, 702

Käppeler, F., Beer, H. \& Wisshak, K. 1989, Reports on Progress in Physics, 52, 945

Koike, C., Wickramasinghe, C., Kano, N., Yamakoshi, K., Yamanoto, T., Kaito, C., Kimura, S. \& Okuda, H. 1995, MNRAS, 277, 986

Lambert, D. L. 1992 Astron. Astrophys. Rev., 3, 201

Lambert, D. L., Gustafsson, B., Eriksson, K. \& Hinkle, K. H. 1986, ApJ Supp., 62, 373

Lewis, R. S. 1992, published in Colangeli, L., Mennella, V., Stephens, J. R. \& Bussoletti, E. $1994, A \& A, 284,583$

Lewis, R.S., Amari, S. \& Anders, E. 1994, Geochim. Cosmochim. Acta, 58, 471

Lewis, R. S. \& Anders, E. 1983, Sci. Am., 549, 54

Lewis, R. S., Anders, E. \& Draine, B. T. 1989, Nature 339, 117

Lewis, R. S., Huss, G. R. \& Lugmair, G. W. 1991, Lunar Planet. Sci., 22, 807 
Lewis, R.S., Tang, M., Wacker, J.F., Anders, E. \& Steel, E. 1987, Nature, 326, 160

Lodders, K. \& Fegley, B. Jr. 1995, Meteoritics, 30, 661

Mutschke, H., Corschner, J., Henning, Th. \& Jäger, C. 1995, ApJ, 454, L157

Nittler, L. R., Alexander, C. M. O'D, Gao, X., Walker, R. M. \& Zinner, E. 1994, Nature, 370,443

Nittler, L. R. et al. 1995, $A p J, 453$, L25

Ott, U. 1993, in Protostars and Planets III, ed. E. H. Levy and J. I. Lunine (Univ. Arizona Press), p. 883

Ott, U. 1996, $A p J, 463,344$

Ott, U., Begemann, F., Yang, J. \& Epstein, S. 1988, Nature, 332, 700

Russel, S. S., Arden, J. W. \& Pillinger, C. T. 1991, Science, 254, 1188

Russel, S. S., Lee, M. R., Arden, J. W. \& Pillinger, C. T. 1995, Meteoritics, 30, 399

Sedlmayr, E. 1994, in IAU Coll. 146: Molecules in the Stellar Environment, ed. U. G. Jørgensen (Springer), p. 163

Virag, A., Zinner, E., Lewis, R. S. \& Tang, M. 1989, Lunar Planet. Sci., 20, 1158

Wdowiak, T. J., Flickinger, G. C. \& Cronin, J. R. 1988, ApJ, 328, L75

Zinner, E. 1995, in Nuclei in the Cosmos III, ed. M. Busso, R. Gallino and C. M. Raiteri, AIP Conf. Proc., 327 , p. 567

Zinner, E., Amari, S., Wopenka, B. \& Lewis, R. S. 1995, Meteoritics, 30, 209

Zinner, E., Tang, M. \& Anders, E. 1989, Geochim. Cosmochim. Acta, 53, 3273

Zinner, E., Wopenka, B., Amari, S. \& Anders, E. 1990, Lunar Planet. Sci., 21, 1379 